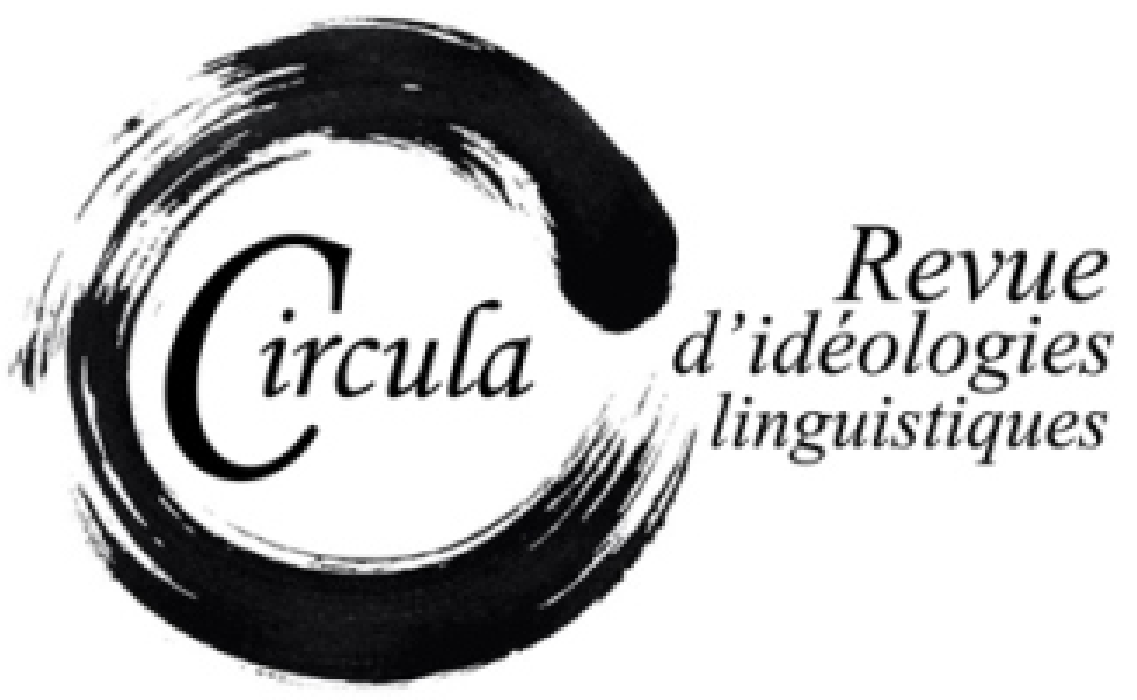

TITRE: PRIMI SPUNTI PER UN'ANALISI DEL DISCORSO SULLA QUESTIONE MERIDIONALE: MATERIALI, METODI, PROSPETTIVE

Auteur: Paolo OrRù, UniversitÀ di Debrecen

Revue: CIRCULA, NUMÉRO 10

PAGES: $22-40$

ISSN: 2369-6761

URI: HTTP://HDL.HANDLE.NET/11143/16772

DOI: HTTPS://DOI.ORG/10.17118/11143/16772 


\section{Primi spunti per un'analisi del discorso sulla questione meridionale: materiali, metodi, prospettive}

Paolo Orrù, Università di Debrecen

paolo.orru@arts.unideb.hu

Riassunto: Il dualismo tra Nord e Sud Italia ha avuto un ruolo importantissimo per la costruzione dell'identità nazionale e ancora oggi struttura gran parte dei discorsi politici e mediatici sullo stato del Paese. È un luogo comune ritenere che, al momento dell'Unità d'Italia, il Paese fosse diviso in un Nord progredito e moderno e un Sud arretrato economicamente e socialmente. Tali costruzioni di senso sono radicate in più ampie correnti di pensiero europee che a cavallo tra XVIII e XIX secolo hanno contribuito a tratteggiare la profonda alterità del meridione europeo e hanno in parte alimentato le imprese risorgimentali italiane. Il presente contributo intende svolgere alcune riflessioni sulle ideologie linguistico-discorsive che hanno alimentato il dualismo Nord-Sud e delineare un percorso di ricerca di lunga durata.

Parole chiave: questione meridionale; analisi del discorso; meridione; dualismo nord-sud; Italia; discorsi politici; Risorgimento

Abstract: The dualism between North and South Italy has played a very important role in the construction of national identity and structures most of the political and media discourse on the state of our country still today. It is commonplace to assume that at the time of the Unification of Italy, the country was divided into an advanced modern North and an economically and socially backward South. Such constructions of meaning are rooted in broader currents of thought that at the turn of the eighteenth and nineteenth centuries have contributed to outlining the profound otherness of the European south and have partly fuelled the Italian Risorgimento. The present paper intends to trace some reflections on the linguistic-discursive ideologies that have nourished the North-South dualism and outlines a long-term research path.

Keywords: southern question; Italy; discourse analysis; north-south dualism; political discourse; Risorgimento 


\section{Nord-Sud: dualismo, potere e discorso ${ }^{1}$}

Salvatore Lupo (2015), uno dei più autorevoli studiosi del Mezzogiorno, in un suo noto volumetto di qualche anno fa dedicato alla questione meridionale decise di inserire un eloquente sottotitolo, che è insieme anche un'idea programmatica: Come liberare la storia del Mezzogiorno dagli stereotipi. L'obiettivo di Lupo è quello di dimostrare come alcuni degli assi portanti della storiografia - da lui definita mainstream - sull'Italia unita siano stati a lungo viziati da eccessive generalizzazioni, basate su letture parziali e, proprio come gli stereotipi² ${ }^{2}$ tramandate acriticamente nel tempo, fino a costruire luoghi comuni ampiamente condivisi anche nel dibattito pubblico e assai difficili da mettere in discussione. Tra tutti è sicuramente centrale il mito dell'arretratezza economica, sociale e culturale del Sud rispetto al Nord. L'opera si inserisce in un più ampio filone di studi ormai trentennale inaugurato da Lupo insieme a Piero Bevilacqua e all'Imes (Istituto meridionale di storia e scienze sociali) che ha trovato spesso sbocco nella rivista Meridiana. Il dibattito scientifico sul tema è certamente ancora aperto e tutt'altro che unanime; tuttavia, l'idea statica, acquisita in decenni di tradizione storiografica, di un meridione omogeneamente immobile, arretrato, fuori dalla modernità, è ormai con convinzione messa ampiamente in discussione ${ }^{3}$. Ciò, ad ogni modo, non significa negare che il Sud al momento dell'Unità non presentasse una moltitudine di problemi e un sostanziale ritardo infrastrutturale e sociale ${ }^{4}$.

La ricostruzione dei dati storiografici è fuori dalle nostre competenze e dagli scopi di questo contributo; quello che ci preme sottolineare, invece, è che tali studi ci indicano una strada per interpretare le dinamiche Nord-Sud, che l'estrema attualità chiama in causa continuamente, sia in merito alla situazione dell'Italia, sia gettando lo sguardo oltre il Mediterraneo.

Gli apporti originali e critici alla materia non si esauriscono nel solo versante delle scienze sociali. Recentemente, si può assistere a un rinnovato interesse verso lo studio del Mezzogiorno da varie angolazioni metodologiche soprattutto nel campo degli studi letterari e culturali. Se è vero che il Sud è stato oggetto continuo di indagine in vari campi delle scienze umane e sociali per tutta la storia

1. Un ringraziamento è dovuto a László Pete, Marco Pignotti e Carmine Pinto per i preziosi commenti e consigli sul quadro storiografico e per i tanti suggerimenti relativi alla composizione del corpus

2. Ci riferiamo qui alla natura cognitiva dello stereotipo, come meccanismo di sovrageneralizzazione di una credenza, che acquisisce nel tempo il valore di senso comune in una comunità (Moscovici, 1981).

3. Luciano Cafagna parla ad esempio di una "modernità passiva", con riferimento alla mancanza di una coesione degli impulsi modernizzatori e di una élite che li aggregasse (Cafagna, 1988). Francesco Barbagallo (2013) preferisce parlare di "questione italiana", per mettere al centro le molteplici spinte disgregatrici in atto sia da Nord sia da Sud; inoltre, ricorda come il divario economico del Mezzogiorno rispetto al Settentrione vada inquadrato nell'ottica di un complessivo ritardo di quest'ultimo rispetto alle altre nazioni industrializzate europee. Le differenze esistenti al momento dell'Unità tra le due aree del Paese non parrebbero, dunque, cosi significative come la tradizione le ha sempre rappresentate. A conclusioni simili è giunto anche Guido Pescosolido (2017a), che inoltre mette ben in evidenza le interconnessioni sul fronte economico venutisi a creare dopo l'Unità e il ruolo che il Mezzogiorno ha avuto nell'avvio dell'industrializzazione delle regioni settentrionali.

4. Per una sintetica ma efficace disamina, si veda Pescosolido (2017b) 
unitaria del Paese; è altrettanto corretto sottolineare la sostanziale novità, per lo meno prospettica, degli studi recenti. Ciò che condividono tali variegate esperienze di ricerca sembra essere proprio, da una parte, la volontà di ridiscutere finalmente le basi degli stereotipi che hanno tormentato la storia unitaria; dall'altra parte, di mettere al centro una visione del Mezzogiorno non più in chiave dualistica, come polo opposto a un Nord, ma come soggetto attivo, da osservare per le proprie peculiarità. Sempre Lupo riassume bene tale intenzione nell'introduzione: «il Mezzogiorno va considerato, al pari di un qualsiasi luogo di questo mondo, come un frammento della modernità. E sui modi della sua modernizzazione che occorre confrontarsi» (Lupo, 2015: xii).

Partendo da traiettorie decisamente differenti, anche Luigi Cazzato (2017) si pone un obiettivo simile nel ripercorrere l'influenza della letteratura di viaggio anglosassone nella costruzione dell'identità nazionale italiana. A tal fine, l'elaborazione teorica di alcuni delle voci più influenti del discorso poste de-coloniale, quali Aníbal Quijano (2007), Walter Mignolo (2007) e lain Chambers (2007), diventa lo strumento per leggere più in profondità la formazione storica e culturale dei discorsi egemonici che ancora oggi strutturano il confronto tra il Nord "virtuoso" e il Meridione "colpevole".

Cazzato applica al contesto storico-politico qui in esame il concetto di «colonialità del potere», mutuato da Quijano. Se il colonialismo va giustamente inteso come un ben preciso prodotto storico delle azioni delle potenze europee, la colonialità è piuttosto la costruzione ideale della superiorità dell'Occidente sulle altre nazioni, ciò che di fatto ha alimentato le imprese coloniali, ma che ne supera i motivi e i luoghi storici. Alcuni dei tratti della differenza tra dominatore e dominato vengono applicati a regioni, nazioni, imperi (come quello russo od ottomano) che non possono essere di fatto colonizzati, ma ai quali viene comunque assegnato un marchio di inferiorità o arretratezza:

[...] il potere della colonialità non si esaurisce con la visione razzista dei rapporti tra colonizzati e colonizzatori, ma esso pervade anche l'interno del mondo moderno occidentale. Nello specifico, meccanismi del potere coloniale hanno governato anche i rapporti tra i popoli che avevano un impero (Inghilterra e Francia) e coloro che non ce lo avevano (ancora: Italia, o più: Spagna), scendendo la scala gerarchica (Cazzato, 2017: 29).

In questo quadro, il dualismo Nord-Sud si sovrappone gradualmente a quello più tradizionale tra Est e Ovest5: allo scontro tra barbarie e civiltà, utile a giustificare le imprese coloniali, si aggiunge quello tra progresso e arretratezza, impiegato invece come strumento per la creazione dell'identità europea; la differenza non è più, dunque, unicamente spaziale e geografica ma anche temporale.

Il viaggio degli intellettuali e diplomatici inglesi (ed europei) è anche un viaggio alla ricerca dei confini meridionali del continente, tracciati di volta in volta prima in corrispondenza delle Alpi, poi di Roma e infine di Napoli. I discorsi elaborati in questa chiave avranno un effetto sulla percezione di

5. È impossibile in questo caso non citare almeno Edward Said (1978). 
sé dell'Italia stessa ${ }^{6}$ : in un contesto in cui le province napoletane rappresentavano un oggetto quasi del tutto ignoto, gli intellettuali italiani si confrontano con le ricostruzioni dei viaggiatori stranieri e attraverso questo contatto inizia a filtrare il dualismo tra Nord e Sud.

Il differenziale di potere tra impero e non-impero avrebbe agito, secondo Cazzato, mediante le stesse logiche coloniali impiegate per conquistare le aree esterne all'Europa. La prospettiva offerta da questo campo di studi è senza dubbio affascinante e ricca di suggestioni, ci preme però allo stesso tempo sottolineare che in nessun caso si vuole in questa sede teorizzare una diretta trasposizione degli elementi culturali, storici e politici delle esperienze coloniali alla dinamica tra Nord e Sud Italia. L'immagine del "Sud colonia del Nord", inoltre, ha fatto e ancora fa parte di un revisionismo che senza dubbio non ci appartiene. Ė bene chiarire ciò per evitare scomodi fraintendimenti dettati in gran parte dalla terminologia impiegata. Rimane rilevante ai nostri fini, invece, la centralità dell'elemento discorsivo nella costruzione delle impalcature concettuali che domineranno il confronto tra le due macro-aree della Penisola. Un differenziale che potremmo definire in questo caso discorsivo tra chi è in grado di imporre un discorso egemonico e chi, invece, finisce per subirlo. Con una metafora militaresca, Cazzato sostituisce alle "cannonate" fisiche impiegate per sottomettere i popoli colonizzati, le più astratte, ma non meno efficaci

[c]annonate della conoscenza che hanno portato chi le ha subite a una sorta di auto-coIonizzazione, fino a considerare per esempio il proprio presente, la propria modernità non all'altezza di tale parola: la modernità è sempre quella degli altri (Cazzato, 2017: 30).

Ma facciamo un passo indietro. II 2011 ha visto un fiorire di iniziative editoriali e scientifiche in occasione del $150^{\circ}$ anniversario dell'Unità; tra queste merita sicuramente un cenno in questa sede l'Atlante culturale del Risorgimento (Banti et al., 2011): organizzato come un lessico del linguaggio politico dal Settecento all'Unità, il volume si propone di riassumere e illustrare sinteticamente alcuni dei lemmi fondamentali alla base della costruzione culturale del Risorgimento. La lista dei vocaboli, divisi in quattro sezioni (percezione del tempo storico, campi dell'esperienza, soggetto nazionale, immaginario istituzionale), si apre con decadenza. La sensazione di una perdita di rilevanza culturale, letteraria, scientifica si fa strada sul finire del XVII secolo ${ }^{7}$ negli scritti dei viaggiatori francesi e olandesi, che ne rintracciavano le cause nell'eccessiva ingerenza della Chiesa nella vita degli italiani e nella dominazione spagnola. Tali impressioni ben presto vengono assorbite anche dagli intellettuali italiani e assumono il carattere di un vero e proprio topos.

6. Cf. De Seta (2014).

7. Del resto, già Fernand Braudel in un suo fondamentale studio ricordava che dalla metà del 1600 in poi «il Mediterraneo si posiziona in modo saldo al di fuori della corrente storica dominante, la quale per secoli l'aveva visto padrone quasi assoluto» (1992: 79). 
Decadenza divenne la parola chiave di ogni analisi della realtà della penisola e di ogni possibile discorso degli italiani sull'Italia: un discorso che dal riconoscimento del distacco della penisola dai paesi considerati ad un livello più alto di sviluppo civile, sociale e culturale voleva individuare le vie di una possibile rinascita - risorgimento; riforma (Verga, 2011: 7).

E la stessa prospettiva interna è ormai in qualche modo viziata: in questa fase lo sbilanciamento egemonico verso nuove potenze che si affacciano all'orizzonte è ormai in atto. II discorso italiano oscillerà tra l'accettazione del modello borghese e liberista delle nazioni settentrionali e la piccata difesa incentrata sulla rivendicazione del primato delle arti e della cultura italiana, di cui il giobertiano Del primato morale e civile degli italiani (Gioberti, 1848) rimarrà l'esempio più fulgido; sarà, tuttavia, la prima di queste due opzioni a prevalere. In questo senso, la rappresentazione della decadenza ha anche un effetto indubbiamente propulsivo e di stimolo culturale: la discesa delle truppe napoleoniche e l'intreccio con i valori illuministici aprono poi nei pensatori italiani l'opportunità per riannodare il rapporto con l'élite d'oltralpe. Col passare degli anni però «le accuse [...] di decadenza, di corruzione, di debolezza, di passività politica e morale rivolte all'Italia» si fecero «sempre più frequenti e dure» (Venturi, 1973: 999).

Nell'area degli studi culturali, Nelson Moe (2004) ha ricostruito l'evoluzione dei discorsi sull'Italia del periodo che va dal 1750 al 1850 in ambito letterario (e non solo) e il loro effetto sui pensatori italiani e sul processo risorgimentale. Partendo anch'egli dall'analisi della letteratura di viaggio, Moe ripercorre il doppio binario su cui scorre l'immagine del Paese ${ }^{8}$ : da una parte la rievocazione della sua grandezza storica, della sua ricchezza culturale, di cui i nuovi imperi (soprattutto quello inglese) vogliono presentarsi come i legittimi eredi, contrapposta continuamente alla miseria, alla sciatteria e allo stato di abbandono coevo. L'Italia diviene niente più che un museo a cielo aperto, in cui passeggiare tra le splendide rovine di un passato glorioso. Dall'altra parte, invece, si sviluppa un'immagine positiva della penisola attraverso il canale del pittoresco. Le bellezze naturali e il clima mediterraneo vengono però via via contrapposte ai caratteri morali degli italiani; tra tutti il più noto è indubbiamente quello dell'“indolenza”.

Le contrapposizioni, cui si è fatto riferimento, sarebbero rimaste ben salde, ma avrebbero interagito con un nuovo modo di concepire il tempo e lo spazio, il quale poneva le regioni occidentali e centrali del continente alla guida del progresso umano, sempre più misurato in termini di benessere materiale e progresso tecnologico (Moe, 2004: 28).

Secondo Moe, insomma, lo sguardo interno verso l'Italia e il Meridione si è alimentato e in parte viene plasmato proprio dal discorso impostato dalle nascenti nazioni che, sulla scorta di una visione teleologica della storia, dividono il continente tra progresso e barbarie, al fine di alimentare e giu-

8. Sul versante linguistico si vedano invece Serianni (2002) e Banfi (2017).

9. Interessante rilevare come tale tratto distintivo fosse specifico dell'Oriente, prima ancora che del Mediterraneo. Ancora più in generale, la pigrizia e l'indolenza sono tratti stereotipici di molte rappresentazioni razzializzanti contemporanee (Reisigl e Wodak, 2001). 
stificare le proprie imprese coloniali e il proprio primato. La volontà di recuperare il divario con le potenze imperiali non è altro che l'accettazione stessa della propria inferiorità, che sconfina progressivamente verso l'assimilazione supina dei valori borghesi del benessere economico e del progresso nord-occidentale. Parole come virtù, civiltà, progresso, opposte a barbarie, indolenza, arretratezza struttureranno il rapporto polarizzante tra Nord e Sud d'Europa, scaveranno un solco nella coscienza dei patrioti (meridionali e non) esuli oltre Manica, supportati dalla diplomazia britannica prima e dall'opinione pubblica poi.

Sarebbero ancora tanti gli esempi di studi, iniziative, progetti più o meno recenti o in corso, purtroppo non è questa la sede in cui è possibile offrire un esaustivo stato dell'arte sulle analisi delle traiettorie delle rappresentazioni del Sud e della costruzione delle sue identità culturali ${ }^{10}$. Si tratta di un campo assai fecondo e si direbbe dai "confini", questi sì, assai larghi e permeabili.

Le vicende storiche che hanno strutturato il dualismo Nord-Sud anticipano l'Unità, l'attraversano e la superano: dall'età moderna alla globalizzazione, passando per la Rivoluzione francese, il Risorgimento, l'esperienza coloniale. Così come molteplici sono i fatti sociali che hanno dato forma all'identità nazionale ${ }^{11}$ : I'unificazione linguistica; la grande emigrazione esterna ed interna; l'esperienza del fascismo; il boom economico e infine l'immigrazione e la nuova grande crisi economica e istituzionale di fine Novecento e inizio XXI secolo. Proprio tali ultimi fatti ci richiamano ancora una volta a una dialettica Nord-Sud di ordine nuovo, o forse, rinnovato: il Sud del Mediterraneo guarda all'Italia e all'Europa come a un Nord carico di speranze e possibilità; viceversa, dall'altra sponda si scorge invece nell'Africa un elemento ansiogeno ${ }^{12}$, per un'invasione che non si è mai veramente realizzata ${ }^{13}$ se non nell'immaginario pubblico, come hanno raccontato le indagini demoscopiche degli ultimi anni.

La crisi economico-finanziaria originatasi negli Stati Uniti nel 2007/2008 e la successiva crisi del debito sovrano nel 2011 hanno riportato prepotentemente alla luce l'armamentario di stereotipi sui paesi mediterranei dell'Unione, sintetizzati nell'acronimo spregiativo Pigs, che se in una fase iniziale comprendeva anche la decisamente settentrionale Irlanda, si è ben presto ridotto a identificare unicamente Portogallo, Italia, Grecia e Spagna. La retorica moralizzante, inoltre, assunta dagli stati del

10. Vale la pena però menzionare almeno Lombardi-Diop e Romeo (2012); Contarini et al. (2018) e il recente numero di Narrativa curato da Marras e Pias (2017).

11. Concetto sicuramente assai sfuggente e precario, problematico e fuorviante, tuttavia, affascinante e onnipresente nei discorsi sullo stato delle nazioni.

12. Ci si consenta a tal riguardo di rimandare almeno al volume in cui si affronta proprio la trattazione mediatica della questione migratoria recente (Orrù, 2017).

13. Nel momento in cui scriviamo, le stime sulla popolazione straniera residente in Italia registrano una percentuale di circa l'8\% sul totale, in linea con il resto del continente, di cui circa la metà è di origine europea. Impossibile allo stato attuale, invece, definire il numero dei cosiddetti irregolari, le ipotesi più recenti parlano di circa 530mila persone (Ismu, 2019). 
Nord all'insegna dell'austerità ${ }^{14}$ da imporre ai paesi "spreconi" ${ }^{15}$ del Sud, altro non è che una versione aggiornata della visione egemonica del Nord a cui si faceva prima riferimento.

Per tornare, però, alla natura di questo contributo e ai suoi (limitati) scopi, quello che ci proponiamo di fare in questa sede è sondare un possibile percorso di studi che vada alla ricerca delle radici degli stereotipi discorsivi sulla "questione meridionale", o sul dualismo Nord-Sud, che dir si voglia, nelle indagini conoscitive, nei racconti sullo stato delle regioni meridionali, nelle corrispondenze e nei dibattiti parlamentari all'indomani dell'Unità. L'obiettivo è quello di offrire una riflessione critica che metta al centro il discorso, inteso nella sua duplice accezione di lingua in uso, cara alla linguistica, e di stratificazione e addensamento di pratiche linguistiche su un determinato fenomeno sociale, impiegata invece dalle scienze sociali, influenzate soprattutto dal pensiero foucaultiano e post-strutturalista.

Gli eventi recenti e le migrazioni ci interrogano sempre più sull'identità nazionale italiana, sempre ammesso che essa possa, o debba, essere veramente definita, ma viviamo un capitolo delle vicende collettive del Mediterraneo da cui è impossibile chiamarsi fuori, anche solo per ovvi motivi di vicinanza geografica, come studiosi e parte attiva della nostra società. Per affrontare questa sfida è però indispensabile provare a rileggere un momento significativo della storia moderna, nel momento in cui certe formazioni discorsive hanno preso corpo. E se da un lato sono stati mossi già passi importanti in altri campi delle scienze umane, l'analisi linguistica ci sembra ancora assente da questo genere di riflessioni critiche sui testi.

Nelle prossime pagine proveremo, dunque, ad offrire una piccola ricognizione dei periodi, dei testi e degli autori da sondare per avviare questo percorso di ricerca.

\section{Materiali e metodi per un'analisi della questione meridionale}

Prima di procedere a indicare una pur sommaria messe di testi e autori da indagare, sarà utile fornire una scansione temporale ragionata dei periodi da osservare. Come anticipato nell'introduzione, infatti, si tratta di almeno tre secoli di storia nazionale, dal XVIII al XX. Va da sé che ognuno di questi può essere poi ulteriormente frazionato e scandagliato più in profondità. Ci limiteremo qui a una prima suddivisione di massima, seppur grezza, assolutamente indispensabile per rendere maneggevole la domanda di ricerca.

14. Si veda a tal proposito l'analisi delle metafore della crisi economica su un corpus di articoli italiani e tedeschi in Ceffa e Rovere (2015).

15. Per citare solo due esempi recenti si pensi ancora alle dichiarazioni rilasciate alla Frankfurter Allgemeine Zeitung nel marzo del 2017 dell'allora Presidente dell'Eurogruppo Jeroen Dijsselbloem sul Sud Europa che "non può spendere tutti i soldi per alcol e donne e poi chiedere aiuto" (https://www.faz.net/aktuell/wirtschaft/wirtschaftspolitik/nach-interview-in-der-f-a-z-dijsselbloem-ich-bedauere-dass-es-als-nord-gegen-sued-aufgefasst-wurde-14937857.html) o al titolo di Der Spiegel del 24 maggio 2018: Die Schnorrer von Rom ovvero "Gli scrocconi di Roma". 
Il periodo pre-risorgimentale che va dal 1750 al triennio giacobino è fondamentale per la formazione di un'identità europea incentrata sul dualismo tra Nord e Sud del continente. Inoltre, l'esperienza dell'llluminismo e della Rivoluzione saranno cardinali per l'emersione del nazionalismo moderno. Come abbiamo accennato poco sopra, è questo il periodo in cui le descrizioni dell'Italia da parte dei viaggiatori stranieri iniziano a farsi più consistenti e allo stesso tempo più dure.

Molti storici individuano l'inizio della fase risorgimentale con la discesa della napoleonica Armata d'Italia nella Penisola, che diede l'avvio al cosiddetto "triennio giacobino" e a un profondissimo rimescolamento degli assetti politici e istituzionali esistenti. Come ricorda Banti, poi, «fu in quel periodo che alcuni ambienti politico-intellettuali formularono per la prima volta chiari progetti di costruzione di uno stato unitario, intorno ai quali si tentò anche di mobilitare energie e individui» (Banti, 2004: XII). La concitata fase segnata da ripetuti tentativi rivoluzionari che culminerà con le due guerre di indipendenza italiane e con il traguardo dell'Unità è per ovvi motivi quella più rilevante ai nostri fini; essa può essere estesa fino ai primi decenni successivi all'Unità, che vedranno da un lato una serie di iniziative importanti ${ }^{16}$ per il Mezzogiorno e una convulsa fase politica - contrassegnata dal trasformismo e dai governi della Sinistra storica - che rischierà a più riprese di porre fine anticipatamente alla neonata nazione.

La scelta per ora è quella di dare la precedenza proprio al periodo risorgimentale e ai primi 50 anni di Unità, prendendo in considerazione, dunque, il XIX secolo fino ai primi anni del Novecento, e dando per assodata la costruzione delle coordinate essenziali di Nord-Sud proposta dai paesi egemoni, soprattutto Inghilterra e Francia. II pensiero intorno alla questione meridionale inizia a delinearsi con nettezza immediatamente prima e dopo l'Unità. I primi 15 anni di esperienza unitaria sono quelli decisivi per l'origine del dualismo tra le due aree del Paese e vedono, soprattutto, lo svilupparsi del pensiero meridionalista attraverso le opere di Pasquale Villari, Leopoldo Franchetti e Sidney Sonnino.

Le ultime due fasi, che qui indichiamo solo in linea generale, corrispondono al Novecento, e più specificamente dalla fine della libertà con l'avvento del fascismo fino alla crisi istituzionale degli anni Novanta, e al primo ventennio degli anni Duemila. II XX secolo ha conosciuto momenti significativi del dualismo Nord-Sud: le migrazioni interne, il boom economico, la fondazione dei movimenti politici regionalisti e indipendentisti (di cui la Lega Nord è solo il caso più conosciuto). La stretta contemporaneità, come già menzionato, ha visto l'emergere di due fenomeni sociali e politici di indubbia salienza come l'immigrazione e il processo sempre più stretto di integrazione europea.

16. Facciamo qui riferimento ad esempio al varo delle leggi speciali per lo sviluppo di alcune regioni (Calabria, Sardegna) e dell'area industriale di Napoli, pensate e avviate da Francesco Saverio Nitti, tra i primi meridionalisti a ricoprire incarichi di governo nel periodo tra il 1911 e il 1920. Per un'agile ricostruzione del ruolo di Nitti e delle sue politiche si veda almeno Barbagallo (2016). 


\subsection{Materiali}

Detto del periodo da noi prescelto per le prime indagini, possiamo ora iniziare a indicare con più precisione una composita tipologia di testi da sottoporre all'analisi linguistica. Si tratta di una ricchissima varietà di generi testuali, che possiamo intanto raggruppare così:

- Testi istituzionali:

- inchieste conoscitive sulla situazione del Mezzogiorno;

- studi e osservazioni;

- dibattiti parlamentari.

- $\quad$ Testi privati e diplomatici:

- memorie;

- epistolari.

- Testi mediali:

- stampa.

In questa sommaria ripartizione si noterà subito l'assenza piuttosto evidente della letteratura, che un indubbio contributo ha dato allo sviluppo delle narrazioni e dei simboli del Risorgimento e alla costruzione dell'identità nazionale, soprattutto in virtù del ruolo del tutto particolare che essa ha rivestito nella storia linguistica del Paese; tuttavia, in questa fase e per meglio servire i nostri scopi ci rivolgeremo a quei testi che per loro natura hanno avuto un effetto diretto sull'azione politica, soprattutto parlamentare.

La questione meridionale e i problemi riscontrati nel Mezzogiorno, come si accennava, rimangono in qualche modo sottaciuti dai primi governi unitari. La tabuizzazione del discorso ha lo scopo di non mettere a rischio la precaria unità raggiunta con fatica e, inoltre, di non dare a vedere alle altre potenze europee le debolezze del Paese. Il passo di seguito, raccontato da Giustino Fortunato, ne dà una plastica esemplificazione:

tornata del 6 aprile 1865 della prima Camera italiana, quando un deputato piemontese, che non altro aveva osato dire se non di scorgere poca simpatia fra le varie regioni del nuovo Regno, dovè smettere tra' rumori e le generali disapprovazioni dell'assemblea! «Prego l'onorevole interpellante», esclamò corrucciato il presidente Cassinis, «di non esternare pensieri che sono altrettanto sconvenienti quanto infondati» \{Bene, bravo!). E il Lamarmora, presidente del Consiglio de' ministri: «Sorgo per protestare contro un'asserzione assolutamente contraria al vero, che mi fa credere che l'onorevole Michelini non sia andato più lontano di Moncalieri (Viva ilarità); perché se egli avesse come me viaggiato per le varie province del 
Regno, si sarebbe persuaso che l'Italia è unita molto più degli altri paesi da lungo tempo formati» (Ha ragione! Applausi) (Fortunato, 1911: 314).

Nonostante la tabuizzazione a cui abbiamo accennato, i dibattiti parlamentari dell'epoca possono sicuramente costituire un altro interessante versante di indagine; nella discussione di specifiche questioni contingenti, ad esempio le leggi speciali per la Calabria, la Sardegna, la Sicilia, i piani di industrializzazione di Napoli, ma anche i dibattiti sulle riforme elettorali e l'estensione del suffragio ${ }^{17}$.

La fase immediatamente successiva all'unificazione e all'annessione delle province meridionali fu affrontata minimizzando sostanzialmente i problemi che andavano via via emergendo nel rapporto tra le due aree del Paese. La pesante sconfitta elettorale delle destre al Sud nel 1874 significò una brusca presa di coscienza da parte dell'élite piemontese delle difficoltà di integrare in un quadro unitario e coeso realtà separate da secoli di storia. Si assiste così al fiorire di una serie di relazioni e corrispondenze di viaggio per accertare lo stato dei territori meridionali. Il fine è quello di comprendere meglio la situazione reale per individuare le aree maggiormente problematiche in cui intervenire.

Prende forma un vasto corpus di inchieste parlamentari e non - ma comunque di natura prettamente politica - sulle regioni del Sud. Tra tutte, le più note sono certamente quelle redatte da Leopoldo Franchetti e Sidney Sonnino che hanno trovato sistemazione nel volume La Sicilia nel 1876 (Franchetti e Sonnino, 1925), che comprende i due lavori Condizioni politiche e amministrative della Sicilia e i Contadini in Sicilia. Franchetti avviò tale impresa già nel biennio 1873-74 con i primi viaggi negli Abruzzi e nel Molise e successivamente in Calabria e Basilicata. Tra le prime inchieste vi fu, invece, la relazione preparata da Enrico Pani Rossi sulla Basilicata e pubblicata nel 1868. Altra opera fondamentale è quella del deputato Giuseppe Massari, figura di meridionale "piemontesizzato", che guidò l'inchiesta sul brigantaggio (pubblicata in Pedio, 1983).

Infine, va ricordata la monumentale opera della Inchiesta parlamentare sulle condizioni dei contadini nelle province meridionali e nella Sicilia, realizzata nei primi del Novecento, che in nove volumi raccoglie le relazioni svolte su richiesta del Parlamento sugli Abruzzi e il Molise, sulle Puglie, sulla Campania, sulla Basilicata e le Calabrie e sulla Sicilia. Di tutt'altro taglio è la ben nota trattazione di carattere antropologico-lombrosiano sulla devianza e sulla delinquenza in Sardegna di Alfredo Niceforo (1897 e ripubblicata nel 1977).

Alle inchieste si affiancano vari altri studi e raccolte di osservazioni personali di intellettuali e politici coevi, come, ad esempio, Governo e governati in Italia di Pasquale Turiello (1882) che «si occupò del Mezzogiorno non per capire e alleviare la sua miseria, ma perché il suo malessere sociale e politico era ritenuto il sintomo rivelatore di un vasto processo di disgregazione, che rischiava di rompere il già precario rapporto esistente tra governanti e governati» (Petraccone, 2000: 113), o gli scritti di Michele Torraca (1879) e Nicola Marselli (1882). Su stimolo di Pasquale Villari, anche scrittori come l'inglese

17. Si veda a questo proposito il lavoro di Pignotti (2017). 
Jessie White Mario e Renato Fucini si recarono a Napoli per riportarne le proprie sensazioni in opere come La miseria in Napoli (1877) e Napoli a occhio nudo (1877).

Il discorso politico è tra tutti quello che più ha la capacità di influire sulla realtà sociale e orientare l'opinione pubblica. Come accennato in precedenza, il parlamento unitario è uno dei luoghi più significativi per la formazione fattuale e discorsiva del dualismo. Ma è anche il luogo in cui a lungo, e soprattutto nella fase storica a cui facciamo riferimento, siede una rappresentanza altamente elitaria, espressione del ceto intellettuale e produttivo della nazione, formata e a contatto con le formulazioni discorsive di cui abbiamo ragionato in apertura di questo contributo. È nel parlamento che vengono prese le decisioni che saranno al centro delle rivendicazioni meridionaliste e dove vengono richieste e istituite le commissioni di inchiesta sullo stato delle province meridionali. È sempre in parlamento che prende vita la questione meridionale, affrontata inizialmente come una mera questione amministrativa e di ordine pubblico ${ }^{18}$, ma che dopo le tornate elettorali del 1874 e '76 diventerà una questione politica centrale fino ai giorni nostri ${ }^{19}$. Il portale storico della Camera dei deputati rende sicuramente agevole il reperimento di un vastissimo corpus di resoconti stenografici digitalizzati dal 1848 al 2018. Si tratta in questo caso di una mole di testi assai significativa, che richiede certamente un'ampia fase di ricerca, segmentazione e recupero dei dati.

Muovendo verso un'altra tipologia testuale, gli epistolari privati dei protagonisti politici dell'epoca e dei parlamentari si dimostrano una fonte interessante per confrontare il discorso pubblico con quello privato e rivelare aspetti ideologici e stereotipi condivisi dall'élite liberale. Da alcuni primi riscontri e dal confronto con la letteratura specializzata, l'epistolario di Cavour20 può rappresentare un buon punto di partenza; sembrerebbe essere, infatti, ricco di missive con alcuni tra i suoi più stretti collaboratori contenenti pareri, descrizioni, suggerimenti sullo stato delle cose nelle province napoletane. Tali scambi hanno svolto tra l'altro anche una funzione fortemente direttiva e hanno contribuito a orientare l'azione del governo. Un'analisi linguistico-discorsiva sul corpus del biennio ‘60-61 potrebbe in tal senso offrire nuovi interessanti sviluppi.

Altro oggetto di indagine tra i testi che abbiamo definito privati possono essere le memorie e i diari. Alcuni esempi, ricavati dalle fonti storiografiche ${ }^{21}$, sono le memorie, poi pubblicate, di alcuni ex garibaldini, come Da Quarto al Volturno di Giuseppe Cesare Abba²2, I Mille di Giuseppe Bandi o le Lettere garibaldine di Ippolito Nievo. Nei testi affiorano di tanto in tanto giudizi sprezzanti sullo stato delle province napoletane e sugli usi della popolazione. Ma le rappresentazioni stereotipiche del

18. Un primo sondaggio sul periodo tra marzo e dicembre del 1861 è stato svolto in Orrù (2019).

19. Per una ricostruzione della nascita della questione meridionale nel parlamento unitario si vedano Sagrestani (2014) e Pignotti (2019).

20. Le versioni integrali degli anni 1860 e '61 sono state pubblicate dall'editore Leo Olschki rispettivamente nel 2005 e nel 2008.

21. Cf. Petraccone (2000).

22. Ripubblicato da Sellerio nel 2010. 
Mezzogiorno possono essere rintracciate in testi apparentemente meno significativi come Le tribolazioni di un insegnante di Ginnasio di Placido Cerri²3, in cui un giovane insegnante piemontese viene mandato a prendere servizio a Bivona, piccola località della Calabria, e ne descrive le condizioni di totale sussistenza e inciviltà.

Infine, non possono essere esclusi da una ricerca di questo genere i testi mediali, e nello specifico la stampa. Del resto, l'opera fondamentale per l'avvio del meridionalismo, Le lettere meridionali di Villari, era il frutto proprio di una corrispondenza giornalistica. Pur nella precarietà dei mezzi, spesso artigianali, nelle ristrettezze imposte dalla censura dei governi locali e con l'enorme ostacolo dell'analfabetismo diffuso in tutta la penisola ${ }^{24}$, il periodo risorgimentale ha visto una notevole espansione delle pubblicazioni e ha contribuito alla creazione di uno spazio di discussione pubblica per gli intellettuali. Oltre ad essere lo strumento ufficiale di diffusione delle notizie politiche e delle novità legislative (attraverso le gazzette), i periodici del tempo ospitavano interventi e corrispondenze sul processo di unificazione e sulle difficoltà incontrate nel governo delle nuove province. Solo per fare un esempio, sui giornali torinesi, come la Gazzetta di Torino o la Gazzetta del popolo, appaiono nei primi mesi del 1861 numerosi articoli critici sulle popolazioni napoletane e sulla loro indole, così come più avanti fu data ampia copertura all'esplosione del brigantaggio. Tali fonti sono tanto più rilevanti se si considera lo strettissimo legame intrattenuto con il governo.

L'elenco di fonti qui fornito è da considerarsi meramente esemplificativo. Data l'estrema varietà e quantità di testi potenzialmente oggetto di analisi, l'inventario proposto non può che essere ancora parziale, in continuo ampliamento e soggetto a futura revisione. Tuttavia, nonostante le difficoltà imposte dalla ricchezza di tipologie testuali, essa è necessaria per poter valutare a pieno la forza e il radicamento degli stereotipi sul Mezzogiorno e l'effetto cumulativo nel loro potenziale presentarsi nelle medesime forme in contesti discorsivi differenti.

\subsection{Analisi del discorso}

Rimangono, infine, da delineare i metodi di analisi per una ricerca di così ampio raggio. La tradizione ormai assestata in Europa dell'analisi del discorso sarà senza dubbio il cardine dell'indagine. Il legame tra produzione linguistica e contesto d'uso nella sua accezione più ampia, includendo le forme dell'interazione, le conoscenze enciclopediche, gli aspetti cognitivi. Gli obiettivi dell'indagine che abbiamo prospettato nei paragrafi precedenti, però, ci inducono allo stesso tempo ad adottare una prospettiva non semplicemente descrittiva, di osservazione degli strumenti linguistici nel loro contesto situazionale e funzionale, ma che sia anche critica $^{25}$ e metta in relazione gli usi della lingua

23. Per una lettura più approfondita in questo senso rimandiamo a Stefanelli (2017).

24. Si rimanda a Serianni (1989: 33-ss) per una ricostruzione del ruolo della stampa nella diffusione e nell'evoluzione dell'italiano, e a Masini (1994) per una dettagliata analisi linguistica.

25. E della sua variante più impegnata, ovverosia l'analisi critica del discorso, la quale si configura più come una prospettiva e un movimento culturale che non una metodologia o un approccio sistematico (Van Dijk, 2001: 353-354). 
con il suo farsi veicolo di trasmissione di ideologie. Esse vanno intese qui non nella loro accezione comune di sistemi concettuali alla base delle varie formazioni politiche. Ci rifacciamo piuttosto alla teorizzazione delle ideologie come profonde e strutturate forme di «socially shared belief systems» elaborata da Teun Van Dijk (2013). Tale specificazione è utile a separare il funzionamento cognitivo delle ideologie dalla loro effettiva applicazione quotidiana, è parimenti fruttuosa per comprenderne meglio i meccanismi o di analizzarle concretamente nella loro realizzazione discorsiva. La struttura delle ideologie incorpora una serie di categorie fondamentali che orientano le credenze sociali dei gruppi e dei singoli soggetti26.

- Identità: Chi siamo? Chi appartiene al nostro gruppo? Chi sono i membri e chi può entrare nel gruppo?

- Attività: Che cosa dobbiamo fare? Qual è il nostro ruolo nella società?

- Obiettivi: Qual è l'obiettivo delle nostre attività?

- Norme e valori: Quali sono le norme che regolano le nostre attività? Cosa è buono e cattivo per noi?

- Relazioni tra gruppi: Chi sono i nostri amici e chi i nostri nemici?

- Risorse: Quali risorse simboliche o materiali formano le basi del nostro potere (o della sua assenza) e della nostra posizione nella società?

Le ideologie trovano dunque sbocco nel discorso quotidiano, quale che sia il canale, e, quando assumono i caratteri di un discorso egemonico, sedimentano fino a diventare senso comune. Il controllo sulla produzione discorsiva, soprattutto quando invisibile, viene progressivamente incamerato nella conoscenza condivisa, fino alla sua stereotipizzazione. Esattamente come avvenuto ai primordi della questione meridionale, quando l'élite settentrionale (in controllo del discorso) compone un mosaico di rappresentazioni che si consoliderà tanto nell'immaginario collettivo quanto nella successiva storiografia mainstream sull'Unità d'Italia.

L'analisi storico-discorsiva, messa a punto da Reisigl e Wodak, ${ }^{27}$ può offrire al ricercatore gli strumenti teorici e metodologici necessari per ricostruire questo percorso. Essa si basa un quadro comprensivo dei vari livelli linguistici di indagine, corroborati da una puntuale integrazione contestuale di dati storici, sociologici. Il livello macro ha come oggetto le condizioni storico-politiche ed economiche dello specifico contesto in cui agiscono e si formano i discorsi in oggetto. A livello intermedio, meso, vengono, invece, selezionate le pratiche discorsive e le tipologie testuali che caratterizzano i testi presi in esame e in accordo, va da sé, con l'obiettivo dell'analisi. Il livello micro consisterà, infine, nell'esame linguistico puntuale dei testi e sulla ricerca degli elementi caratterizzanti delle pratiche discorsive, prendendo in considerazione i vari livelli di analisi del linguaggio (lessico, retorica e metafore, deissi,

26. Nostra traduzione dello schema presentato in Van Dijk (2013: 178).

27. Si veda Reisigl e Wodak (2001). 
sintassi) e le strutture argomentative. Concretamente, per i nostri scopi, il livello macro corrisponderà al periodo storico e politico definito nella sezione precedente (dall'inizio del Risorgimento alla fine del XIX secolo); il livello meso comprenderà principalmente in una prima fase le tipologie testuali delle inchieste, dei dibattiti parlamentari e degli epistolari; il livello micro affronterà un'analisi linguistica il più accurata e comprensiva possibile.

Impresa non semplice, data la veste grafica di molti di questi testi, è quella di coniugare metodi di indagine qualitativa e quantitativa; ci riferiamo soprattutto all'impiego degli strumenti per l'analisi informatica dei testi elaborati nella tradizione della linguistica dei corpora. La ripulitura e sistemazione del corpus si rivelerebbe in certi casi assai complessa e richiederebbe una grande quantità di tempo. Tuttavia, la digitalizzazione e ripubblicazione di alcune fonti permette di combinare senza eccessiva difficoltà la classica analisi linguistica e stilistica con un approccio corpus based (Baker et al., 2008; Partington, 2004). I dati quantitativi e la ricerca statistica della preminenza linguistico-discorsiva di lessemi, collocazioni e costrutti sintattici in un corpus di testi omogenei per tipologia testuale o semplicemente semantica possono offrire chiavi di lettura che la sola intuizione personale potrebbe tralasciare. Ciononostante, può essere rilevante rimarcare come questo genere di analisi non sia intesa da noi come una sostituzione di metodi di lettura e indagine più tradizionali, ma vada considerato piuttosto un utile strumento integrativo (Biber, Conrad et Randi, 1998: 7-8). L'approccio quantitativo da solo, infatti, non può essere sufficiente a esaurire un'analisi linguistico-discorsiva.

\section{Considerazioni finali}

L'obiettivo del presente contributo è stato quello di avviare una riflessione che avvicini gli studi linguistici a una sempre più ampia bibliografia critica sulla questione meridionale. Riteniamo che il dualismo tra Nord e Sud del Paese sia uno dei temi sociali e politici più rilevanti nella storia italiana; la sua presenza attraversa l'intera storia unitaria fino ai giorni nostri, in cui il divario economico tra le due aree non fa che ampliarsi progressivamente.

La "questione" però non può essere ricondotta al solo caso italiano e alle sue vicende storiche, si inserisce in correnti di pensiero ben più ampie che hanno iniziato ad agire nei decenni precedenti al Risorgimento e che ancora oggi influenzano le dinamiche internazionali tra Nord e Sud del mondo.

Il programma di ricerca qui delineato, ancora in fieri, si intende aperto a modifiche e integrazioni, e trova spazio nel quadro degli studi sulle ideologie linguistiche, intese come manifestazioni linguistico-discorsive di sistemi cognitivi più ampi, costituiti da idee, stereotipi, atteggiamenti sui fenomeni extralinguistici. Attraverso la pervasiva presenza in tipologie testuali particolarmente salienti dal punto di vista della creazione e del mantenimento di posizione egemoniche, alcune forme linguistiche filtrano nel dibattito pubblico quotidiano, assumendo via via la caratura di conoscenza stereotipica condivisa e diventando vere e proprie costruzioni di senso comune. 
In un periodo storico come quello recente, in cui la posizione e il ruolo dell'Italia nel "mare di mezzo" sono tornati di estrema attualità, un'indagine ad ampio raggio sulla formazione dei discorsi che hanno strutturato la percezione odierna del dualismo Nord-Sud può apparire di sicura utilità epistemologica. 


\section{Bibliografia}

Abba, Giuseppe Cesare (2010), Da Quarto al Volturno, Palermo, Sellerio.

Baker, Paul et al. (2008), "A Useful Methodological Synergy? Combining Critical Discourse Analysis and Corpus Linguistics to Examine Discourses of Refugees and Asylum Seekers in the UK Press», Discourse and Society, vol. 19, n’3, p. 273-306.

Banfi, Emanuele (2017), Italiano e altre varietà italo-romanze in Europa e nel Mediterraneo nel secolo XIX, Firenze, Franco Cesati.

Banti, Alberto Mario (2004), Il Risorgimento italiano, Roma-Bari, Laterza.

Banti, Alberto Mario et al. (eds.) (2011), Atlante culturale del Risorgimento. Lessico del linguaggio politico dal Settecento all'Unità, Roma-Bari, Laterza.

Barbagallo, Francesco (2013), La questione italiana: il Nord e il Sud dal 1860 a oggi, Roma-Bari, Laterza.

Barbagallo, Francesco (2016), «Nitti e il Mezzogiorno tra politica ed economia», in Sabino Cassese (ed.), Lezioni sul meridionalismo. Nord e Sud nella storia d'Italia, il Mulino, Bologna, p. 103-114.

Biber, Douglas, Conrad, Susan et Randi Reppen (1998), Corpus Linguistics: Investigating Language Structure and Use, Cambridge, Cambridge University Press.

Braudel, Fernand (1992), Civilization and Capitalism, 15-18th Century, Los Angeles, University of California Press.

Cafagna, Luciano (1988), «Modernizzazione attiva e modernizzazione passiva», Meridiana, 2, p. 229240.

Cavour, Camillo (2005), Epistolario. Vol. XVII (1860), Firenze, Leo Olschki.

Cavour, Camillo (2008), Epistolario. Vol. XVIII (1861), Firenze, Leo Olschki.

Cazzato, Luigi (2017), Sguardo inglese e Mediterraneo italiano. Alle radici del meridionismo, Milano, Mimesis.

Ceffa, Sabina e Giovanni Rovere (2015), «La crisi economica europea nella stampa tedesca e italiana. questioni metodologiche di analisi discorsiva», Studi italiani di linguistica teorica e applicata, vol. 44, n², p. 199-226.

Chambers, Iain (2007), Le molte voci del Mediterraneo, Milano, Raffaello Cortina.

Contarini, Silvia et al. (eds.) (2018), Da ieri a oggi. Tragitti del Sud nella cultura italiana contemporanea, Firenze, Franco Cesati.

De Seta, Cesare (2014), L'Italia nello specchio del Grand Tour, Milano, Rizzoli.

Fortunato, Giustino (1911), Il Mezzogiorno e lo stato italiano. Discorsi politici (1880-1910), vol. II, Bari, Laterza. 
Franchetti, Leopoldo e Sidney Sonnino (1925), Inchiesta in Sicilia, Firenze, Vallecchi.

Fucini, Renato (1921), Napoli a occhio nudo, Firenze, Vallecchi.

Gioberti, Vincenzo (1848), Del primato morale e civile degli italiani, Napoli, Stabilimento Tip. e Calc. di C. Batelli e comp.

Ismu (2017), Ventiquattresimo rapporto sulle migrazioni (2018), Milano, Franco Angeli.

Lombardi-Diop, Cristina e Caterina Romeo (eds.) (2014), L'ttalia postcoloniale, Firenze, Le Monnier.

Lupo, Salvatore (2015), La questione. Come liberare la storia del Mezzogiorno dagli stereotipi, Roma, Donzelli.

Marras, Margherita e Giuliana Pias (eds.) (2017), Nuove frontiere del Sud Genesi e sviluppo di un pensiero plurale sul Sud nella letteratura e nella cultura dell'ttalia contemporanea, Narrativa, vol. 39.

Marselli, Nicola (1882), La politica dello Stato italiano, Napoli, Morano.

Masini, Andrea (1994), «La lingua dei giornali dell'Ottocento», in Luca Serianni e Pietro Trifone (eds.), Storia della lingua italiana, vol. II: Scritto e parlato, Torino, Einaudi, p. 635-665.

Mignolo, Walter (2007), «Delinking. The rhetoric of modernity, the logic of coloniality and the grammar of de-coloniality», Cultural Studies, vol. 21, n² 2-3, p. 449-514.

Moe, Nelson (2004), Un paradiso abitato da diavoli. Identità nazionale e immagini del Mezzogiorno, Napoli, L'ancora del Mediterraneo.

Moscovici, Serge (1981), «On Social Representation», in Joseph P. Forgas (ed.), Social Cognition: Perspectives on Everyday Understanding, London, Academic Press, p. 181-210.

Niceforo, Alfredo (1977), La delinquenza in Sardegna: note di sociologia criminale, Cagliari, Edizioni della Torre.

Orrù, Paolo (2017), Il discorso sulle migrazioni nell'Italia contemporanea: un'analisi linguistico-discorsiva sulla stampa (2000-2010), Milano, Franco Angeli.

Orrù, Paolo (2019), «Leggere la questione meridionale alle sue origini: analisi linguistica e discorsiva dei dibattiti parlamentari nell'Italia postunitaria», in Id. (ed.), Vecchie e nuove questioni: il dualismo Nord-Sud in epoca contemporanea, Firenze, Franco Cesati, p. 105-132.

Partington, Alan (2004), «Corpora and Discourse, a Most Congruous Beast», in Alan Partington, John Morley and Louann Haarman (eds.), Corpora and Discourse, Bern, Peter Lang, p. 11-20.

Pedio, Tommaso (1983), Inchiesta Massari sul brigantaggio, Manduria, Lacaita.

Petraccone, Claudia (2000), Le due civiltà. Settentrionali e meridionali nella storia d'Italia dal 1860 al 1914, Roma-Bari, Laterza.

Pignotti, Marco (2017), La moltitudine apolitica. Culture politiche e voto alle masse in età giolittiana (1904-1913), Firenze, LeMonnier. 
Pignotti, Marco (2019), «Dall'inchiesta Franchetti-Sonnino sulla Sicilia ai dibattiti parlamentari: il Mezzogiorno nel dibattito pubblico dell'Italia postunitaria», in Paolo Orrù (ed.), Vecchie e nuove questioni: il dualismo Nord-Sud in epoca contemporanea, Firenze, Franco Cesati, p. 195-204.

Quijano, Anibal (2007), «Coloniality and Modernity/Rationality», Cultural Studies, vol. 21, n² 2-3, p. 168-178.

Reisigl, Martin e Wodak, Ruth (2001), Discourse and discrimination: Rhetorics of Racism and AntiSemitism, London and New York, Routledge.

Sagrestani, Marco (2014), «La questione meridionale nel dibattito parlamentare della prima legislatura unitaria», in Gabriele Paolini (ed.), La prima emergenza dell'Italia unita. Brigantaggio e questione meridionale nel dibattito interno e internazionale nell'età della destra storica, Firenze, Polistampa, p. 51-78.

Said, Edward (1978), Orientalism, London, Penguin.

Serianni, Luca (1989), Il primo Ottocento, Bologna, II Mulino.

Serianni, Luca (2012), «Lingue e dialetti d'Italia nella percezione dei viaggiatori sette-ottocenteschi», in Id., Viaggiatori, musicisti, poeti. Saggi di storia della lingua italiana, Milano, Garzanti, p. 55-88.

Stefanelli, Diego (2017), «Le Tribolazioni di un insegnante di Ginnasio di Placido Cerri», in Stefano Lazzarin e Agnès Morini (eds.), Maitres, précepteurs et pédagogues. Figures de l'enseignant dans la littérature italienne, Bern, Peter Lang, p. 83-100.

Torraca, Michele (1879), I meridionali alla Camera, Napoli, De Angelis.

Turiello, Pasquale (1882), Governo e governati in Italia, Bologna, Zanichelli.

Van Dijk, Teun Adrianus (2001), «Critical Discourse Analysis», in Deborah Schiffrin, Deborah Tannen and Heidi Hamilton (eds.), Handbook of Discourse Analysis, Oxford, Blackwell, p. 352-371.

Van Dijk, Teun Adrianus (2013), «ldeology and Discourse», in Michael Freeden, Lyman Tower Sargent and Marc Stears (eds.), The Oxford Handbook of Political Ideologies, Oxford, Oxford University Press, p. 175-196.

Venturi, Pietro (1973), «L'Italia fuori d'Italia», in Ruggiero Romano e Corrado Vivanti (eds.), Storia d'Italia, vol. III, Torino, Einaudi, p. 987-1481.

Verga, Marcello (2011), «Decadenza», in Alberto Mario Banti, Antonio Chiavistelli, Luca Mannori e Marco Meriggi (eds.), Atlante culturale del Risorgimento. Lessico del linguaggio politico dal Settecento all'Unità, Roma-Bari, Laterza, p. 5-18. 